\title{
High degree of genetic diversity of non-polio enteroviruses identified in Georgia by environmental and clinical surveillance, 2002-2005
}

\begin{abstract}
Correspondence
N. Khetsuriani

nck7@cdc.gov
\end{abstract}

Received 9 June 2010

Accepted 23 July 2010

\author{
N. Khetsuriani, ${ }^{1}$ T. Kutateladze, ${ }^{2}$ E. Zangaladze, ${ }^{2}$ T. Shutkova, ${ }^{2}$ \\ S. Peñaranda, ${ }^{1}$ W. A. Nix, ${ }^{1}$ M. A. Pallansch ${ }^{1}$ and M. S. Oberste ${ }^{1}$ \\ ${ }^{1}$ Division of Viral Diseases, Centers for Disease Control and Prevention, Atlanta, GA, USA \\ ${ }^{2}$ National Center for Disease Control and Public Health, Tbilisi, Georgia
}

\section{INTRODUCTION}

Genetic characterization of enterovirus strains has become the basis for current enterovirus classification. Sequence analysis of enterovirus genomes has been used to determine the current molecular-based criteria for defining enterovirus serotypes, leading to identification of an increasing number of new enteroviruses (Pallansch \& Roos, 2001). In addition, phylogenetic studies are useful for establishing geographical and temporal relationships between enterovirus strains, thus providing information important for outbreak investigations and epidemiological studies. Supplementing traditional approaches to enterovirus surveillance (virus culture with subsequent serotyping in neutralization reactions or immunofluorescence assays

Abbreviations: AFP, acute flaccid paralysis; CDC, Centers for Disease Control and Prevention; WHO, World Health Organization.

The GenBank/EMBL/DDBJ accession numbers for the partial VP1 sequences determined in this study are GQ914812-GQ914903. with monoclonal antibodies) with molecular methods of enterovirus typing allows timely detection of the emergence of new strains, which are often associated with large-scale outbreaks (Mullins et al., 2004; Wikswo et al., 2009).

Despite the increasing availability of enterovirus sequences from various parts of the world, substantial geographical gaps remain, primarily with regard to regions outside the USA and western Europe, due to limited or non-existent enterovirus surveillance in these areas. In the Republic of Georgia, enterovirus surveillance has been conducted primarily as part of poliovirus surveillance and includes environmental and human components. Surveillance based on human specimens is very limited and mainly includes testing of specimens collected through acute flaccid paralysis (AFP) surveillance, occasional testing of patients with meningitis or meningoencephalitis, and limited special stool surveys usually based on convenience samples. To supplement the existing surveillance, limited but more 
systematic environmental surveillance is being conducted in various regions of Georgia.

To describe non-polio enteroviruses circulating in Georgia and to determine their temporal and geographical relationships with previously characterized strains from other regions, we conducted molecular characterization of non-polio enteroviruses isolated from environmental and human specimens in Georgia during 2002-2005 and compared their nucleotide sequences with previously characterized enterovirus strains.

\section{METHODS}

Biological specimens. Environmental specimens (sewage water; $n=44$ ) were collected by grab-type sampling. Water specimens were collected in four to six regions per year, for a total of seven different regions throughout the country (Fig. 1, Table 1). These seven regions account for approximately $60 \%$ of Georgia's population. The collections took place between April and November from 2002 to 2005 (nine to 13 collections per year). Water specimens were delivered to the laboratory in Tbilisi, Georgia, on cold packs, within $24 \mathrm{~h}$ after collection. Clinical surveillance included testing AFP cases and, in a less systematic manner, testing specimens from patients with enterovirus-related syndromes, as well as persons with other conditions and healthy individuals. Stool specimens $(n=210)$ were collected during 2003-2005 from various sources, including AFP cases, hospitalized children with various clinical conditions (acute meningitis/meningoencephalitis and syndromes unrelated to enteroviruses) from a major paediatric hospital in Tbilisi, and from healthy children enrolled in stool surveys. Clinical information for the sources of specimens was not recorded, with the exception of AFP cases. The numbers of stool specimens tested in each study year varied from 67 to 73 .

Enterovirus isolation and typing. Initial virus isolation and typing was performed at the National Polio and Enterovirus Laboratory of Georgia, Tbilisi. Stool suspensions were made by adding $2 \mathrm{~g}$ faecal specimen to $10 \mathrm{ml}$ PBS containing $10 \%$ chloroform. After shaking, the stool suspensions were centrifuged for $20 \mathrm{~min}$ at $1500 \mathrm{~g}$ at $4{ }^{\circ} \mathrm{C}$ (WHO, 2004). Environmental specimens were concentrated approximately 100 -fold by a two-phase method prior to culture (WHO, 2003). After adjusting the $\mathrm{pH}$ to 7.2 by adding $\mathrm{NaOH}$ or $\mathrm{HCl}$, samples were centrifuged at $1000 \mathrm{~g}$ for $10 \mathrm{~min}$. Tubes with a pellet were kept overnight at $4{ }^{\circ} \mathrm{C}$. Then, $39.5 \mathrm{ml} 20 \%$ Dextran T40, $287 \mathrm{ml} 29 \%$ PEG-6000 and $35 \mathrm{ml} 5 \mathrm{M} \mathrm{NaCl}$ were added to $500 \mathrm{ml}$ of the sewage supernatant. The mixture was agitated vigorously for $1 \mathrm{~h}$ at room temperature using a horizontal shaker, transferred to a separation funnel and left overnight at $4{ }^{\circ} \mathrm{C}$. After separation, the small bottom phase $(2-3 \mathrm{ml})$ and a few millilitres of the interphase were collected drop-wise, and the pellet was resuspended, treated with chloroform ( $22 \%$ by volume) by shaking for $1 \mathrm{~min}$ and centrifuged, as with the faecal suspensions. Antibiotics were added to the upper, aqueous phase (penicillin and streptomycin to final concentrations of 100 international units $\mathrm{ml}^{-1}$ and $100 \mathrm{mg} \mathrm{ml}^{-1}$, respectively).

Virus isolation was performed according to standard World Health Organization (WHO) procedures, by inoculating $0.1 \mathrm{ml}$ stool suspension into cell-culture tubes or $0.5 \mathrm{ml}$ environmental extract into $75 \mathrm{~cm}^{2}$ cell-culture flasks (WHO, 2003). Two sequential passages (5-7 days) were performed in two cell lines, RD (human rhabdomyosarcoma cells) and L20B (mouse L cells expressing the human poliovirus receptor CD155). If no cytopathic effect was observed on first passage, a second, blind passage was performed. Positive RD specimens were passaged onto L20B cells. Initial typing of isolates was performed by a microneutralization test using intersecting pools of type-specific antisera (National Institute for Public Health and the Environment) (WHO, 2003). Poliovirus isolates were submitted to intratypic differentiation at the WHO Regional Reference Laboratory at the Institute of Poliomyelitis and Viral Encephalitides, Moscow, Russia.

Molecular characterization and typing of non-polio enterovirus isolates. Molecular characterization and typing of the non-polio enterovirus isolates was performed at the Picornavirus Laboratory, Centers for Disease Control and Prevention (CDC), GA, USA. RTPCR with primers targeting a portion of the VP1 region ( 340 nt) followed by amplicon sequencing was used for enterovirus typing (Oberste et al., 2003). Partial VP1 sequences of enterovirus strains from the study were compared with sequences of representative strains available in GenBank or in the CDC enterovirus sequence collection. The sequences were aligned using CLUSTAL_x (Thompson et al., 1997) and phylogenetic relationships were reconstructed using MEGA, version $4.0 \beta$ (Tamura et al., 2007), with the JTT substitution model (Jones et al., 1992) and 1000 bootstrap pseudoreplicates.

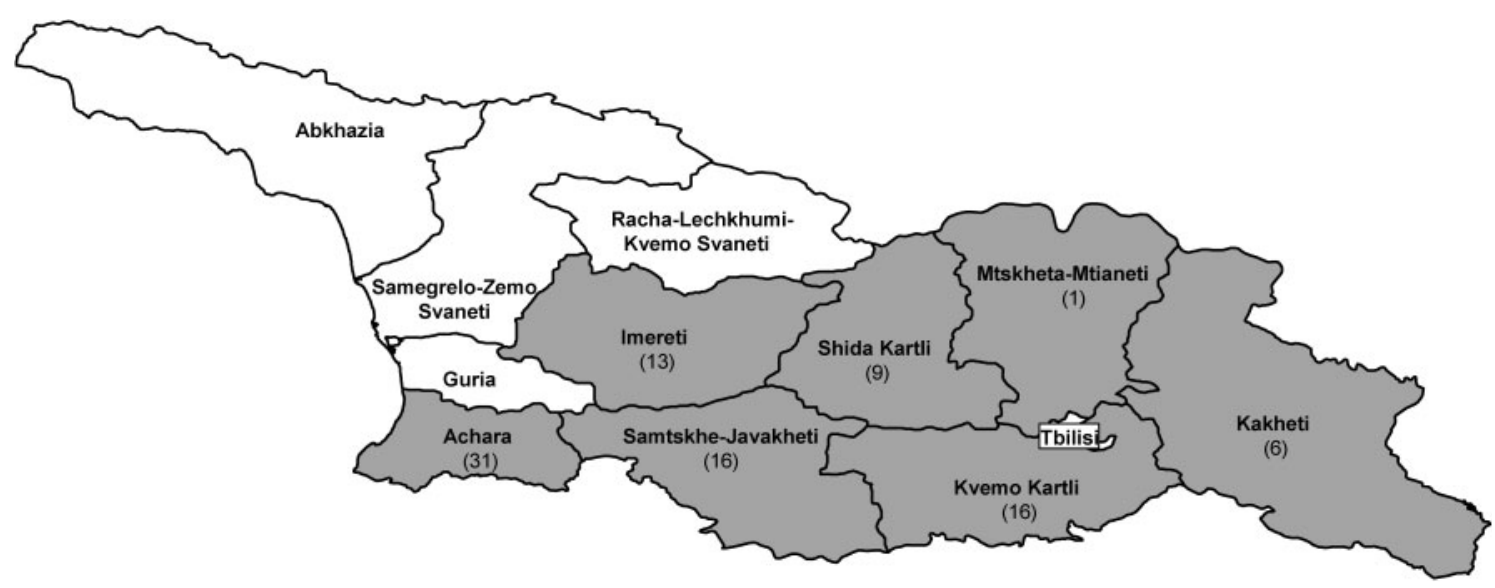

Fig. 1. Geographical distribution of 92 enterovirus strains isolated from environmental specimens in Georgia, 2002-2005. Note that details of the geographical origin of stool specimens were not available. 
Table 1. Non-polio enteroviruses isolated in Georgia, 2002-2005

\begin{tabular}{|c|c|c|c|c|c|c|c|c|}
\hline \multirow[t]{2}{*}{ Enterovirus } & \multicolumn{5}{|c|}{ Number of isolates } & \multirow{2}{*}{$\begin{array}{l}\text { From } \\
\text { sewage }\end{array}$} & \multirow{2}{*}{$\begin{array}{l}\text { From } \\
\text { stool }\end{array}$} & \multirow{2}{*}{$\begin{array}{c}\text { Nt identity to } \\
\text { respective } \\
\text { prototype (\%) }\end{array}$} \\
\hline & 2002 & 2003 & 2004 & 2005 & 2002-2005 & & & \\
\hline \multicolumn{9}{|l|}{ Species HEV-A } \\
\hline CVA10 & 0 & 1 & 0 & 0 & 1 & 0 & 1 & 75.5 \\
\hline \multicolumn{9}{|l|}{ Species HEV-B } \\
\hline CVB2 & 1 & 2 & 0 & 0 & 3 & 3 & 0 & $82.2-84.0$ \\
\hline CVB3 & 1 & 0 & 0 & 0 & 1 & 1 & 0 & 74.0 \\
\hline CVB4 & 0 & 0 & 5 & 2 & 7 & 6 & 1 & $79.9-81.9$ \\
\hline CVB5 & 1 & 0 & 0 & 6 & 7 & 7 & 0 & $76.2-78.9$ \\
\hline $\mathrm{E} 2$ & 0 & 0 & 1 & 0 & 1 & 0 & 1 & 80.2 \\
\hline E3 & 1 & 1 & 5 & 4 & 11 & 10 & 1 & $81.2-83.2$ \\
\hline E6 & 5 & 0 & 0 & 16 & 21 & 18 & 3 & $72.1-76.3$ \\
\hline E7 & 5 & 6 & 0 & 0 & 11 & 11 & 0 & $77.2-79.7$ \\
\hline E11 & 1 & 1 & 2 & 3 & 7 & 7 & 0 & $76.6-83.5$ \\
\hline E12 & 2 & 0 & 0 & 0 & 2 & 2 & 0 & $80.4-82.0$ \\
\hline E13 & 0 & 2 & 4 & 0 & 6 & 5 & 1 & $74.1-78.5$ \\
\hline E14 & 0 & 0 & 2 & 2 & 4 & 2 & 2 & $78.3-79.9$ \\
\hline E19 & 3 & 3 & 0 & 0 & 6 & 6 & 0 & $75.4-80.5$ \\
\hline E20 & 1 & 2 & 8 & 0 & 11 & 7 & 4 & $79.8-81.0$ \\
\hline E24 & 0 & 1 & 1 & 0 & 2 & 2 & 0 & $76.4-79.2$ \\
\hline E25 & 0 & 0 & 0 & 2 & 2 & 2 & 0 & $75.9-78.8$ \\
\hline E29 & 0 & 2 & 0 & 0 & 2 & 2 & 0 & 81.1 \\
\hline E30 & 0 & 6 & 0 & 0 & 6 & 0 & 6 & $75.5-76.6$ \\
\hline E33 & 1 & 0 & 0 & 0 & 1 & 1 & 0 & 76.6 \\
\hline Total & 22 & 27 & 28 & 35 & 112 & 92 & 20 & \\
\hline $\begin{array}{l}\text { Most common } \\
\text { serotypes }\end{array}$ & $\begin{array}{l}\text { E6 }(22.7 \%), \\
\text { E7 }(22.7 \%), \\
\text { E19 }(13.6 \%)\end{array}$ & $\begin{array}{l}\text { E7 }(22.2 \%), \\
\text { E30 }(22.2 \%), \\
\text { E19 }(11.1 \%)\end{array}$ & $\begin{array}{l}\text { E20 }(28.6 \%), \\
\text { E3 }(17.9 \%), \\
\text { E13 }(14.2 \%)\end{array}$ & $\begin{array}{l}\text { E6 }(45.7 \%), \\
\text { CVB5 }(17.1 \%), \\
\text { E3 }(11.4 \%)\end{array}$ & $\begin{array}{l}\text { E6 }(18.8 \%), \\
\text { E3 }(9.8 \%), \\
\text { E7 }(9.8 \%), \\
\text { E20 }(9.8 \%)\end{array}$ & & & \\
\hline
\end{tabular}

\section{RESULTS}

A total of 112 non-poliovirus isolates were obtained from a total of 254 specimens, comprising 92 isolates recovered from 44 environmental samples and 20 isolates recovered from 210 human specimens. Typing by sequencing a fragment of $\sim 340 \mathrm{nt}$ of the VP1 region identified 20 different non-polio enterovirus serotypes: one from human enterovirus species A (HEV-A) and 19 from HEV-B. No new serotypes were detected. The distribution of identified enteroviruses by source of isolation is presented in Table 1. Overall, the most commonly identified enteroviruses during 2002-2005 included echovirus (E) 6 (18.8\% of isolates), E3, E7 and E20 (9.8\% each), coxsackievirus (CV) B4 and CVB5 (6.3\% each), E11 (6.3\%), and E13, E19 and E30 ( $5.4 \%$ each). Three serotypes were isolated from human specimens only, whilst 11 serotypes were recovered from environmental specimens only and six serotypes were isolated from both specimen types (Table 1).

In addition to non-polio enteroviruses, there were 96 poliovirus isolates identified during 2002-2005, including 56 isolates from environmental specimens and 40 isolates from stool specimens. Type 1 poliovirus accounted for 18 isolates, type 2 for 43 and type 3 for 35 . Intratypic differentiation by the Reference Laboratory determined that all poliovirus isolates were vaccine type, consistent with the use of oral polio vaccine for routine childhood immunization in Georgia.

E3 and E11 were detected during each of the 4 study years and E20 was detected during 3 of the 4 years; nine serotypes were detected in 2 of the 4 years, and eight serotypes were detected in only a single year. The predominant serotypes differed over time. During the study period, a total of eight serotypes appeared among the most common enteroviruses for a given year. Of these, E3, E6, E7 and E19 appeared among the three most common serotypes in 2 of the study years, whilst E13, E20, E30 and CVB5 were each common only in a single year.

As expected, the viruses were characterized by substantial divergence from their respective prototype strains. In most cases, nucleotide sequence identity to the prototype was less than $80 \%$ and did not exceed $84 \%$ for any serotype (Table 1). Phylogenetic analysis of the enterovirus strains from Georgia also revealed a high degree of genetic diversity within a serotype. Five serotypes were represented by more than one genetic lineage, including two serotypes (E13 and E3) with three lineages and three serotypes 
(CVB4, CVB5 and E6) with two lineages (Fig. 2). In most cases, different lineages of the same serotype co-circulated in the same year. The only exception was E6, which had one lineage detected only in 2002 and another only in 2005. Some genetic lineages persisted for more than 1 year. For example, an E11 lineage was detected each year during 2002-2005 (Fig. 2). When the same serotype was detected in both environmental and stool samples, the strains were closely related genetically, indicating the lack of substantial differences among strains by source of isolation (Fig. 2).

The closest matches of the study isolates of CVB2, CVB4, E7, E11, E12, E13, E19, E30, E33 and one of the lineages of CVB5 were found among contemporary strains only (within 10 years). These close matches were often from distant geographical regions, including the Middle East, North America, Europe, South Asia and Africa. The isolates of CVB3 and E29 were closely related to both contemporary and earlier strains, whilst the closest matches for E2 and E14 were only the strains isolated in Sweden several decades ago (in 1982). The isolates of E20 and E24, as well as one of the CVB5 lineages, were clearly distinct from previously characterized strains. The Georgian E30 strains from 2003 belonged to the same genetic lineage as some strains identified in countries of the former USSR (Russia, Ukraine, Uzbekistan) during 2004-2005, but were clearly distinct from the E30 strains from these countries and Georgia circulating during 1999-2000 (Lukashev et al., 2008). One of the E6 lineages was most closely linked to the strain detected in 2005 in neighbouring Azerbaijan.

\section{DISCUSSION}

This study revealed a high degree of non-polio enterovirus diversity in Georgia, which is particularly striking for a small country with very limited enterovirus surveillance and a relatively short observation period. Phylogenetic analysis of the isolates demonstrated that many enteroviruses were represented by more than one genetic lineage; some lineages were clearly distinct from previously characterized strains. Of particular interest was the appearance of E3, E19 and E20 among the most common enteroviruses in Georgia. These serotypes are very rarely reported in the multi-year data from regions with wellestablished enterovirus surveillance systems, such as the USA (Khetsuriani et al., 2006), Japan (Infectious Disease Surveillance Center, 2000, 2002a, b, 2008) and some western European countries (Antona et al., 2007; Maguire et al., 1999).

E3 strains from Georgia were characterized by a high degree of genetic diversity. One of the E3 lineages, circulating in Georgia in 2002-2003, clustered in the same lineage as contemporary viruses identified in India and Bangladesh, whilst the other lineage was related to E3 strains identified subsequently in the USA in 2007-2008. According to US enterovirus surveillance data for 19702005 (Khetsuriani et al., 2006), E3 was among the most commonly isolated serotypes in the 1970s, but has become very rare since that time (Khetsuriani et al., 2006; Morens et al., 1979). Similarly, E3 has rarely been reported in England (data from 1975-1994; Maguire et al., 1999), Japan (1982-2008) (Infectious Disease Surveillance Center, 2000, 2002a, b, 2008), France (2000-2004) (Antona et al., 2007), Malaysia (1992-2002) (Saraswathy et al., 2004) and Tunisia (1992-2003) (Bahri et al., 2005), as well as in other places (Richter et al., 2006). Therefore, the appearance of E3 among the predominant serotypes in Georgia, identification of co-circulating lineages of E3 in different geographical regions and the first reported recent outbreak due to E3 (an aseptic meningitis outbreak in South Africa in 2003; Yeats et al., 2005) suggest that this serotype might be re-emerging as a common enterovirus. Unfortunately, the VP1 sequences of the viruses from the South Africa outbreak were not available for comparison.

E19, another virus commonly detected in Georgia, has been reported rarely or not at all by most surveillance systems (Antona et al., 2007; Khetsuriani et al., 2006; Richter et al., 2006; Saraswathy et al., 2004). The only exception is a high level of E19 activity in England in 1975, when it was the most common serotype identified in both clinical and environmental specimens (Maguire et al., 1999; Sellwood et al., 1981). The E19 strains from Georgia comprised a single lineage. Their closest matches were the E19 isolates from Oman identified in 1999, but the analysis of phylogenetic relationships was limited by the small number of available comparison sequences.

E20 isolates from Georgia clustered together in a single genetic lineage, but very few sequences were available for comparison. Historically, E20 has been very rare in most published surveillance system reports (Antona et al., 2007; Khetsuriani et al., 2006; Richter et al., 2006; Saraswathy et al., 2004), but it was identified relatively commonly in Tunisia during 1992-2003 (Bahri et al., 2005) and among neonates in the Netherlands during 1993-1995 (VerboonMaciolek et al., 2002), accounting for approximately $7 \%$ of all isolates in each study.

Distinct genetic lineages of enteroviruses in Georgia probably result in part from substantial gaps in enterovirus surveillance both regionally and worldwide, and in part from the introduction of new strains from other regions. The relatedness of several enterovirus lineages from Georgia to contemporary strains from distant geographical regions, including those linked to large-scale outbreaks (e.g. E13 and E33, both in 2000-2001; Huang et al., 2003; Mullins et al., 2004), demonstrates the ability of enterovirus lineages to spread over large geographical areas and underlines the importance of enterovirus surveillance worldwide. However, due to substantial gaps in currently existing surveillance and the common asymptomatic nature of enterovirus infection, it is very difficult to identify the precise geographical origins of strains.

Temporal and geographical comparisons of the study strains with previously characterized enteroviruses were 
(a)

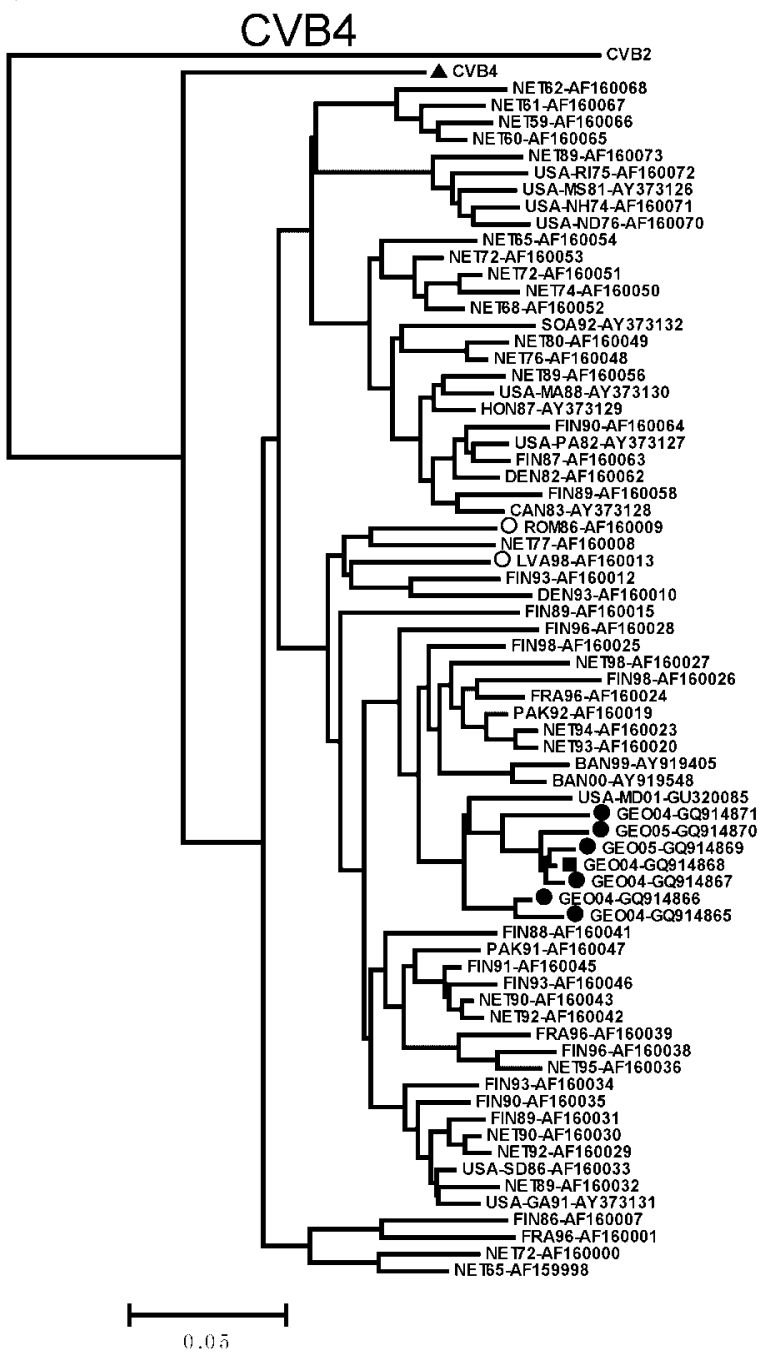

(c)

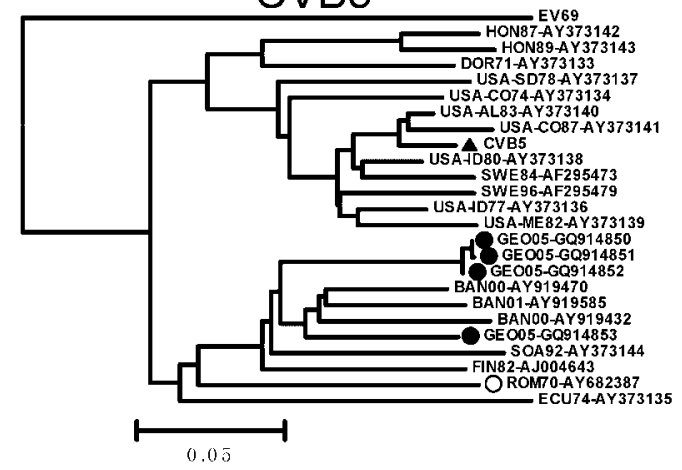

(b)

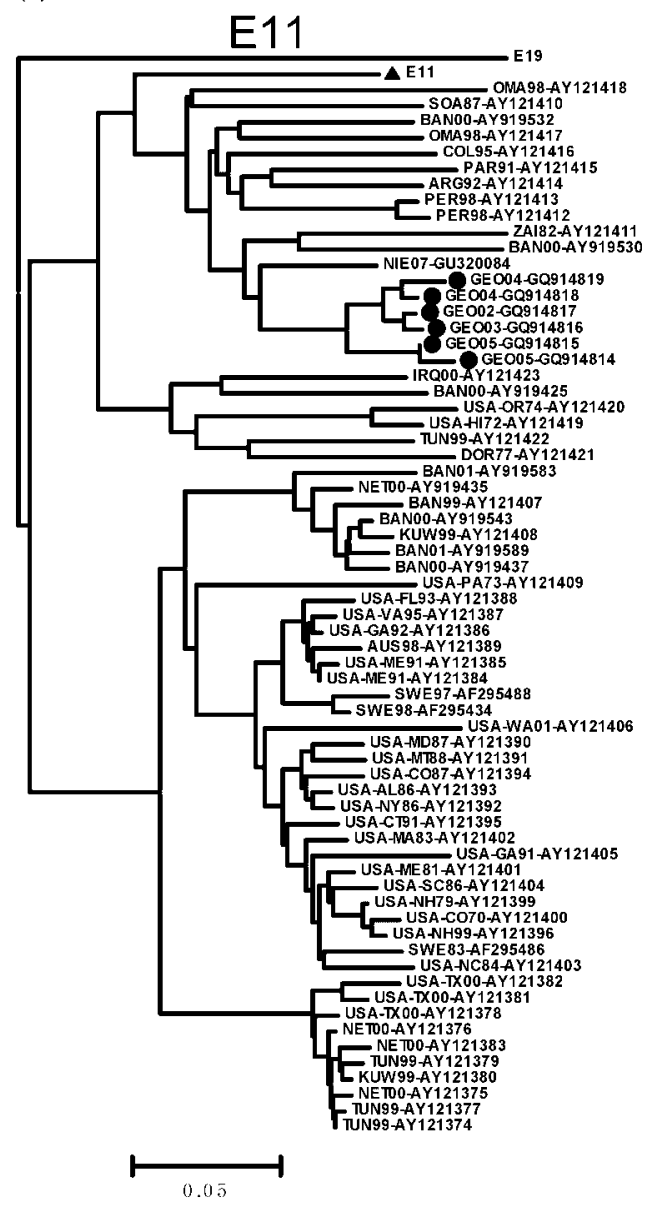

(d)

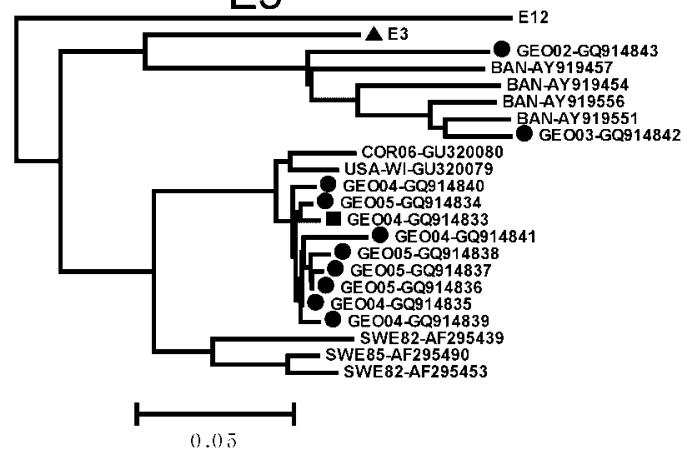

Fig. 2. Phylogenetic relationships of selected enterovirus serotypes with enterovirus sequences available in GenBank and the CDC enterovirus sequence collection (partial VP1 sequences), Georgia, 2002-2005. (a) CVB4; (b) E11; (c) CVB5; (d) E3; (e) E6; (f) E7; (g) E13; (h) E30. Phylogenetic trees were constructed from alignments of partial VP1 sequences for each serotype by the neighbour-joining method implemented in CLUSTAL_X. •, Study strains from environmental specimens, Georgia; $\mathbf{\square}$, study strains from stool samples, Georgia; $\square$, strains from other studies, Georgia; $\bigcirc$, enterovirus strains from neighbouring countries; $\boldsymbol{\Delta}$, enterovirus prototypes. CV, Coxsackievirus; E, echovirus; EV, enterovirus. Bars, 0.05 nucleotide substitutions per site. 
(e)

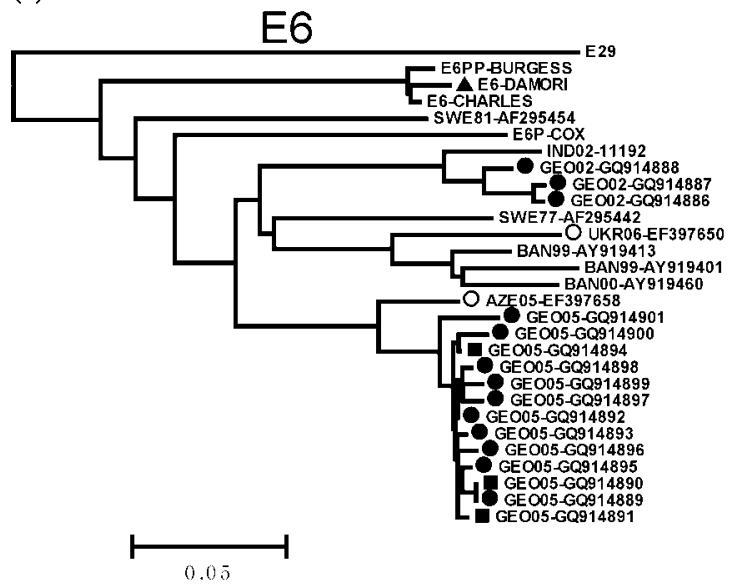

(g)

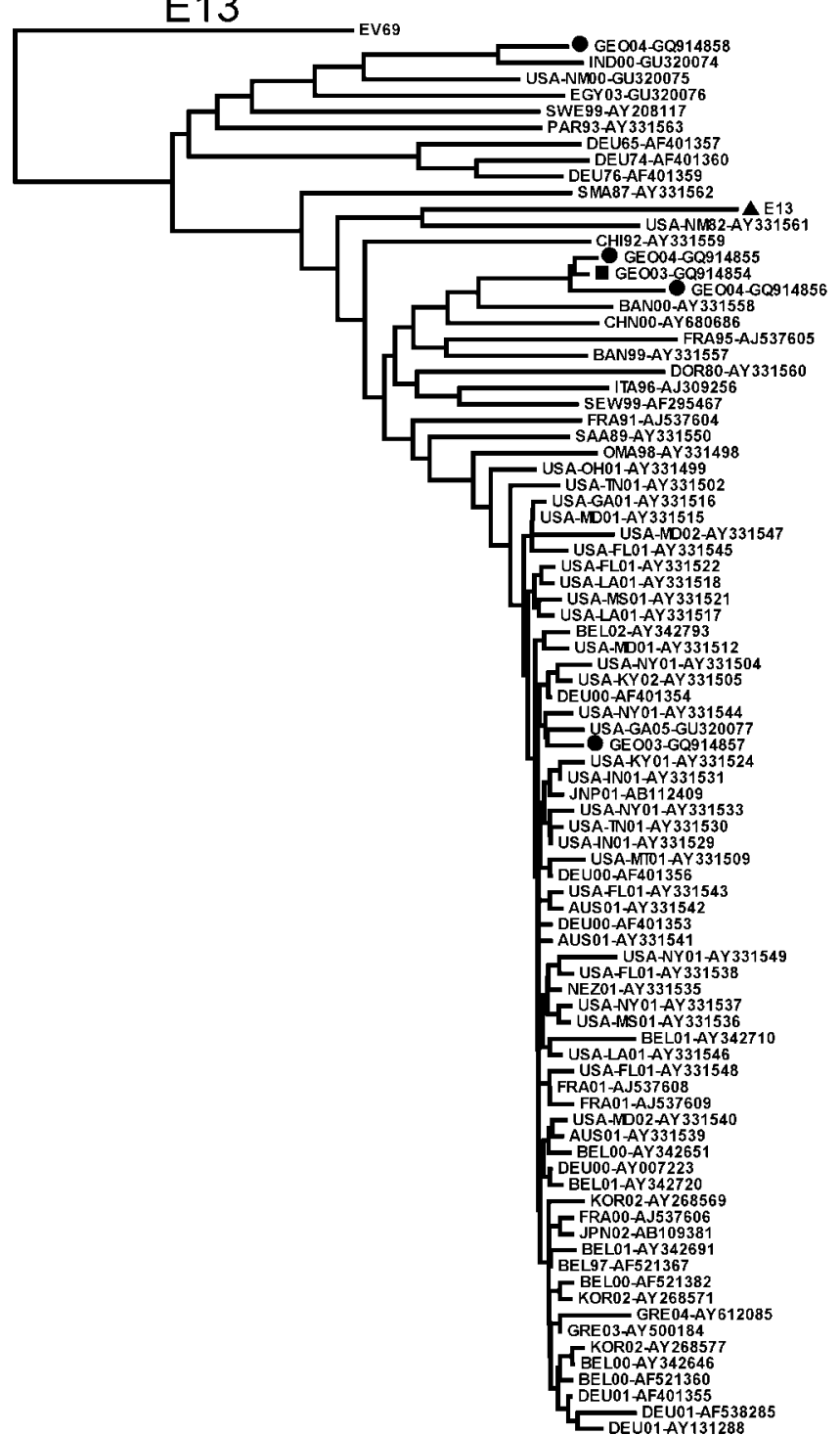

(f)

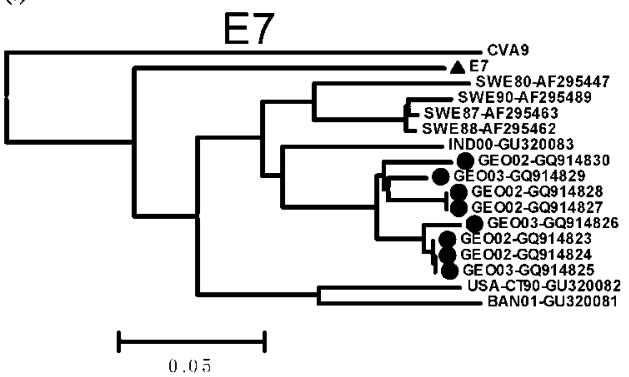

(h)

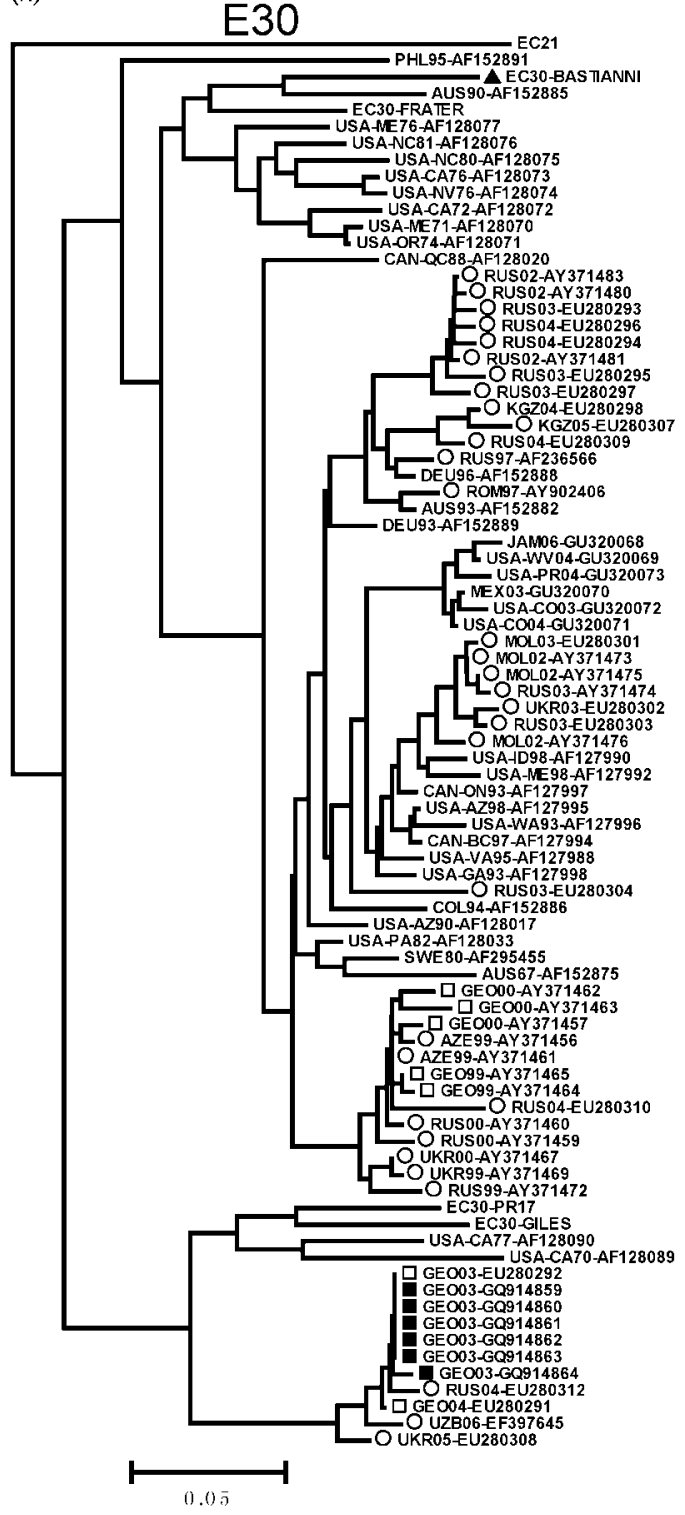


restricted by the availability of comparison sequences, particularly for relatively rarely identified serotypes. With the exception of one report on the molecular epidemiology of E30 in countries of the former Soviet Union (Lukashev et al., 2008), almost no comparison sequences were available from the immediate surrounding region (Turkey, Armenia, Azerbaijan, southern Russia, Romania, Iran, Ukraine, Moldova, Turkmenistan, Uzbekistan, Kazakhstan and Kyrgyzstan). The information on the range of enterovirus serotypes circulating in Georgia and their genomic sequences provided by the present study contribute substantially to the database of circulating enterovirus strains. These baseline data can be used in the future for determining temporal and geographical relationships of enteroviruses, which is particularly useful for outbreak investigations and for detecting the emergence of new genetic lineages of enteroviruses.

As predominant serotypes change over time and the emergence of new genetic lineages of enteroviruses in a particular area is often associated with large-scale outbreaks, continued monitoring of enterovirus strains is needed. The present study demonstrated the added value of environmental surveillance for enteroviruses. Enterovirus surveillance in Georgia, as in many other areas, has been driven by the needs of the Global Polio Eradication Initiative (WHO, 2003, 2004). In such settings, non-polio enteroviruses are frequently isolated in the process of laboratory surveillance for poliomyelitis but are rarely characterized beyond differentiating them from polioviruses, resulting in substantial gaps in enterovirus surveillance globally. The usefulness of environmental surveillance and a good correlation with surveillance data based on clinical specimens has been demonstrated previously (Sedmak et al., 2003; Sellwood et al., 1981). Despite its modest scale, the environmental surveillance in Georgia substantially complemented the clinical surveillance for enteroviruses. Without it, many serotypes detected in environmental samples only would have been missed, and are probably missed in other parts of the world. Nevertheless, the present study is likely to have underestimated the true diversity of enteroviruses in Georgia, as, similar to many other countries, it relies on a protocol and cell lines aimed at maximizing chances of poliovirus detection rather than detecting the broadest possible range of enteroviruses. In addition, many enteroviruses, particularly CVA from HEV-A, do not grow well in cell culture and are usually underdetected by traditional cell-culture-based surveillance systems. This has been changing lately in some countries (e.g. the USA) with the wider introduction of molecular methods of enterovirus typing, which provide more accurate information on circulating enteroviruses (Khetsuriani et al., 2006).

Existing enterovirus surveillance systems are very diverse with regard to objectives, specimen entry points, geographical and population coverage, and laboratory methods. Expanding the scale of enterovirus surveillance with greater standardization of both laboratory and epidemiological components is needed to further improve the accuracy and timeliness of monitoring geographical and temporal patterns of enterovirus circulation. In Georgia, expanding surveillance to include all regions and initiating systematic collection of clinical data for enteroviruses originating from human specimens will improve the monitoring of enterovirus-related clinical syndromes.

In summary, a broad range of circulating non-polio enteroviruses with a very high degree of genetic diversity was identified in Georgia. Epidemiological and molecular data from the present study established the relationships of enteroviruses from Georgia with previously characterized strains and confirmed the importance of both clinical and environmental surveillance for enteroviruses. In order to monitor circulating enteroviruses effectively, particularly in settings with limited clinical surveillance, systematic environmental surveillance should be encouraged.

\section{ACKNOWLEDGEMENTS}

This project was supported in part by the International Science and Technology Center (ISTC), through the Biotechnology Engagement Program US Department of State (ISTC Project No. G-1310p). The findings and conclusions in this report are those of the author(s) and do not necessarily represent the views of CDC.

\section{REFERENCES}

Antona, D., Lvque, N., Chomel, J. J., Dubrou, S., Lvy-Bruhl, D. \& Lina, B. (2007). Surveillance of enteroviruses in France, 2000-2004. Eur J Clin Microbiol Infect Dis 26, 403-412.

Bahri, O., Rezig, D., Ben Nejma-Oueslati, B., Ben Yahia, A., Ben Sassi, J., Hogga, N., Sadraoui, A. \& Triki, H. (2005). Enteroviruses in Tunisia: virological surveillance over 12 years (1992-2003). J Med Microbiol 54, 63-69.

Huang, Q. S., Carr, J. M., Nix, W. A., Oberste, M. S., Kilpatrick, D. R., Pallansch, M. A., Croxson, M. C., Lindeman, J. A., Baker, M. G. \& Grimwood, K. (2003). An echovirus type 33 winter outbreak in New Zealand. Clin Infect Dis 37, 650-657.

Infectious Disease Surveillance Center (2000). Enterovirus surveillance in Japan, 1982-1999. IASR 21, 212-213.

Infectious Disease Surveillance Center (2002a). Virus isolation/ detection from aseptic meningitis cases, 1997-2002. Infectious Agents Surveillance Report. http://idsc.nih.go.jp/iasr/prompt/circle-g/meningi/ menin9702e.html

Infectious Disease Surveillance Center (2002b). The trend of enterovirus isolation in association with aseptic meningitis, 19992002. IASR 23, 193-194.

Infectious Disease Surveillance Center (2008). Virus isolation/ detection from aseptic meningitis cases, 2003-2008. Infectious Agents Surveillance Report. http://idsc.nih.go.jp/iasr/prompt/circle-g/meningi/ menin0307e.html

Jones, D. T., Taylor, W. R. \& Thornton, J. M. (1992). The rapid generation of mutation data matrices from protein sequences. Comput Appl Biosci 8, 275-282.

Khetsuriani, N., LaMonte-Fowlkes, A., Oberste, M. S. \& Pallansch, M. A. (2006). Enterovirus surveillance - United States, 1970-2005. MMWR Surveill Summ 55, 1-20. 
Lukashev, A. N., Ivanova, O. E., Eremeeva, T. P. \& Gmyl, L. V. (2008). Analysis of echovirus 30 isolates from Russia and new independent states revealing frequent recombination and reemergence of ancient lineages. J Clin Microbiol 46, 665-670.

Maguire, H. C., Atkinson, P., Sharland, M. \& Bendig, J. (1999), Enterovirus infections in England and Wales: laboratory surveillance data: 1975 to 1994. Commun Dis Public Health 2, 122-125.

Morens, D. M., Zweighaft, R. M. \& Bryan, J. M. (1979). Non-polio enterovirus disease in the United States, 1971-1975. Int J Epidemiol 8, 49-54.

Mullins, J. A., Khetsuriani, N., Nix, W. A., Oberste, M. S., LaMonte, A., Kilpatrick, D. R., Dunn, J., Langer, J., McMinn, P. \& other authors (2004). Emergence of echovirus type 13 as a prominent enterovirus. Clin Infect Dis 38, 70-77.

Oberste, M. S., Nix, W. A., Maher, K. \& Pallansch, M. A. (2003). Improved molecular identification of enteroviruses by RT-PCR and amplicon sequencing. J Clin Virol 26, 375-377.

Pallansch, M. \& Roos, R. (2001). Enteroviruses: polioviruses, coxsackieviruses, echoviruses, and newer enteroviruses. In Fields Virology, 4th edn, pp. 723-775. Edited by D. M. Knipe \& P. M. Howley. Philadelphia, PA: Lippincott Williams and Wilkins.

Richter, J., Koptides, D., Tryfonos, C. \& Christodoulou, C. (2006). Molecular typing of enteroviruses associated with viral meningitis in Cyprus, 2000-2002. J Med Microbiol 55, 1035-1041.

Saraswathy, T. S., Khairullah, N. S., Sinniah, M., Fauziah, M. K., Apandi, M. Y. \& Shamsuddin, M. (2004). Laboratory acute flaccid paralysis surveillance in Malaysia: a decade of commitment to the WHO global polio eradication initiative. Southeast Asian J Trop Med Public Health 35, 421-424.

Sedmak, G., Bina, D. \& MacDonald, J. (2003). Assessment of an enterovirus sewage surveillance system by comparison of clinical isolates with sewage isolates from Milwaukee, Wisconsin, collected August 1994 to December 2002. Appl Environ Microbiol 69, 71817187.

Sellwood, J., Dadswell, J. V. \& Slade, J. S. (1981). Viruses in sewage as an indicator of their presence in the community. J Hyg (Lond) 86, 217-225.

Tamura, K., Dudley, J., Nei, M. \& Kumar, S. (2007). MEGA4: Molecular Evolutionary Genetics Analysis (MEGA) software version 4.0. Mol Biol Evol 24, 1596-1599.

Thompson, J. D., Gibson, T. J., Plewniak, F., Jeanmougin, F. \& Higgins, D. G. (1997). The CLUSTAL_X windows interface: flexible strategies for multiple sequence alignment aided by quality analysis tools. Nucleic Acids Res 25, 4876-4882.

Verboon-Maciolek, M. A., Krediet, T. G., van Loon, A. M., Kaan, J., Galama, J. M. D., Gerards, L. J. \& Fleer, A. (2002). Epidemiological survey of neonatal non-polio enterovirus infection in the Netherlands. J Med Virol 66, 241-245.

WHO (2003). Guidelines for Environmental Surveillance of Poliovirus Circulation. Geneva: World Health Organization.

WHO (2004). Polio Laboratory Manual, 4th edn. Geneva: World Health Organization.

Wikswo, M. E., Khetsuriani, N., Fowlkes, A. L., Zheng, X., Penaranda, S., Verma, N., Shulman, S. T., Sircar, K., Robinson, C. C. \& other authors (2009). Increased activity of coxsackievirus B1 strains associated with severe disease among young infants in the United States, 2007-2008. Clin Infect Dis 49, e44-e51.

Yeats, J., Smuts, H., Serfontein, C. J. \& Kannemeyer, J. (2005). Investigation into a school enterovirus outbreak using PCR detection and serotype identification based on the $5^{\prime}$ non-coding region. Epidemiol Infect 133, 1123-1130. 